dimensional control of eye movements, and aspects of treatment of nystagmus and updating concepts of the operations of different classes of eye movements. Also new to this edition is a CD-ROM that provides figures, tables, and over 60 videos referenced to the text. The CD-ROM also contains the text so that readers can peruse it on their computer screen or absorb it in the conventional paper format in an armchair. Aficionados of standard text may prefer the print layout edition, but the CD-ROM affords the ability to jump from physiology to a cross-referenced clinical section and back again at the click of a mouse. Short video clips are particularly helpful to the chapter on central eye movement disorders. However, the full text CD-ROM version fills only a third of the screen and is not comfortable to read.

The text is divided into two parts. Part I deals with the physiology of eye movement systems and provides a comprehensive discussion relevant to physiologists, but also pertinent to clinicians who will find it helpful and in some instances necessary to understanding Part II. Moreover this section discusses both clinical and laboratory methods of eye movement examination. Each chapter contains a summary that is an extremely helpful learning tool.

The second part comprises about half of the book and covers peripheral palsies and strabismus, beginning with anatomical and physiological aspects of the orbit, muscles and peripheral nerves. Then central eye movement disorders are discussed.

The book is extensively referenced and up-to-date. The authors have built on the strength of the two prior editions to make this the most comprehensive, current and useful textbook available on eye movements. Fundamental and clinical neuroscientists as well as practicing neurologists will find the Neurology of Eye Movements ideal as a reference source and a pleasure to read as a learning medium. The videos and figures on the CD-ROM will bring the text to life on their desks.

James A. Sharpe Toronto, Ontario

\section{Diagnosis and Treatment of Parkinson's Disease - State of} THE ART. 1999. Edited by H. Przuntek, T. Muller. Published by Springer Wien New York. 220. C\$166.60 approx.

Diagnosis and Treatment of Parkinson's Disease - State of the Art is the collection of papers from a 1997 workshop of the same title in Budapest, Hungary. The authors are experts in the field, both clinicians and basic scientists. As it represents the topics under discussion at the meeting, this book does not cover all aspects of diagnosis and treatment. It focuses on current theories of Parkinson's disease pathophysiology and etiology with comprehensive discussions of candidate genes, possible environmental factors and the role of oxidative stress. Pathological correlates of the main clinical features of Parkinson's disease are discussed in detail.

Regarding treatment, there is a clear and well-organized discussion of pharmacologic strategies for managing advanced Parkinson's disease. Other papers are highly specialized, with extensive coverage of the putative mechanisms and efficacy of budipine as a novel antiparkinson agent. Potential ways of controlling oxidative damage are also thoroughly covered.

Surprisingly, intermixed are useful reviews on multiple system atrophy and the differential diagnosis and phenomenology of tremor.

The majority of this text provides very specialized information which is best suited to experts in movement disorders who have a strong research interest, particularly basic science research. The papers are thorough presentations of their individual topics and are supplemented by extensive referencing. Inevitably, advances in the field since 1997 are not included in the text. However, the vast majority of the information included is still topical and relevant to today's research

Connie Marras

Toronto, Ontario

Parkinson's disease and Movement Disorders. Diagnosis and Treatment Guidelines for the Practicing Physician. 2000. Edited by Charles H. Adler, J. Eric Ahlskog. Published by Humana Press. 480 pages. C $\$ 187.00$ approx.

This text discusses all of the common and most of the uncommon movement disorders in a well-organized fashion. It covers, as the name suggests, clinical features, diagnostic testing and treatment and does not attempt to comprehensively discuss other aspects of the disorders such as pathology, pathophysiology or epidemiology. Mention is made of important points in these areas for the more common disorders, however. Functional neuroimaging of movement disorders is not covered in detail, but diagnostic techniques routinely used in clinical practice are discussed reliably.

Parkinson's disease is covered in greatest detail. A comprehensive discussion of clinical features, differential diagnosis and treatment are discussed for early and advanced disease, including the latest advances in surgical treatments and transplantation. For most of the other movement disorders a phenomenological approach is taken, which allows for a unified discussion of the approach to diagnosis of most clinical syndromes encountered in movement disorder practice. Treatment options are described explicitly, with clear recommendations on which approaches are likely to be most useful. The authors and editors are careful to make the discussions internationally relevant, with mention of treatments available either in Europe or North America. Useful tables are provided to aid quick reference in diagnosis and treatment. The editors and many of the chapter authors are internationally known in the field of movement disorders and are well-qualified to provide these reviews.

The writing is clear and concise. There is little redundancy and excellent cross-referencing between chapters for ease of information retrieval. Useful general references are provided at the end of each chapter; there is not extensive referencing of specific information provided in the discussions.

This book is best suited to general neurologists with an interest in treating movement disorders, or for fellows training in movement disorders. It does not assume prior knowledge, and provides a detailed and logical approach to the diagnosis and differential diagnosis of all of the common movement disorders from the most basic of principles to more sophisticated points.

Connie Marras

Toronto, Ontario 